ISSN 1855-3966 (printed edn.), ISSN 1855-3974 (electronic edn.)

\author{
ARS MATHEMATICA CONTEMPORANEA 21 (2021) \#P2.05 \\ https://doi.org/10.26493/1855-3974.2351.07b \\ (Also available at http://amc-journal.eu)
}

\title{
Density results for Graovac-Pisanski's distance number
}

\author{
Lowell Abrams \\ University Writing Program and Department of Mathematics, \\ The George Washington University, Washington, DC 20052, USA \\ Lindsey-Kay Lauderdale * \\ Department of Mathematics, Towson University, Towson, MD 21252, USA
}

Received 2 June 2020, accepted 13 May 2021, published online 30 October 2021

\begin{abstract}
The sum of distances between every pair of vertices in a graph $G$ is called the Wiener index of $G$. This graph invariant was initially utilized to predict certain physico-chemical properties of organic compounds. However, the Wiener index of $G$ does not account for any of its symmetries, which are also known to effect these physico-chemical properties. Graovac and Pisanski modified the Wiener index of $G$ to measure the average distance each vertex is displaced under the elements of the symmetry group of $G$; we call this the Graovac-Pisanski (GP) distance number of $G$. In this article, we prove that the set of all GP distance numbers of graphs with isomorphic symmetry groups is dense in a half-line. Moreover, for each finite group $\Gamma$ and each rational number $q$ within this half-line, we present a construction for a graph whose GP distance number is $q$ and whose symmetry group is isomorphic to $\Gamma$. This construction results in graphs whose vertex orbits are not connected; we also consider an analogous construction which ensures that all vertex orbits are connected.
\end{abstract}

Keywords: Wiener index, distance number, Graovac-Pisanski index, graph automorphism group, chemical graph theory.

Math. Subj. Class. (2020): 05C12, 05C25, 05C35, 05C92

\footnotetext{
*Corresponding author. The author is the Jess and Mildred Fisher Endowed Professor of Mathematics in the Fisher College of Science and Mathematics at Towson University and is partially supported by this endowment.

E-mail addresses: labrams@gwu.edu (Lowell Abrams), llauderdale@towson.edu (Lindsey-Kay Lauderdale)
} 


\section{Introduction}

Throughout this article, all graphs considered are simple and finite, and all groups considered are finite. We let $V(G)$ and $E(G)$ denote the vertex set and edge set of a graph $G$, respectively. The Wiener index of $G$ is the sum of all distances between pairs of vertices in $G$, namely

$$
W(G):=\frac{1}{2} \sum_{u \in V(G)} \sum_{v \in V(G)} d(u, v),
$$

where $d(u, v)$ is the length of a shortest path between $u$ and $v$ in $G$. This graph invariant was original defined by Wiener [14], where he considered graphical representations of molecules. In particular, each vertex in $V(G)$ represents an atom of a molecule and each edge in $E(G)$ represents a bond between atoms. Wiener [14] used this graph invariant to establish an equation that predicts the boiling points of paraffin molecules.

Other physico-chemical properties of organic molecules, including refractive index, heat of isomerization, heat of vaporization, density, surface tension, viscosity, and chromatographic retention time, were later linked to the Wiener index [5]. Consequently, the Wiener index of classes of compounds, including benzenoids [6], chains [13], and trees [2], were calculated; Mohar and Pisanski [11] described numerous algorithms that compute the Wiener index of a graph in general. An interested reader can see [10] and the references within for more results on this graph invariant.

The symmetries of molecules are known to effect certain physico-chemical properties of organic compounds [12]. In this article, we are interested in a modification of $W(G)$ that accounts for these symmetries of $G$. Recall the set of adjacency-preserving permutations of $V(G)$ is called the automorphism group of $G$ and is denoted by Aut $G$. Graovac and Pisanski [4] defined the distance number of $G$ to be the average

$$
\delta(G):=\frac{1}{|\operatorname{Aut} G||V(G)|} \sum_{u \in V(G)} \sum_{\sigma \in \operatorname{Aut} G} d(u, \sigma(u)) .
$$

We call this invariant the Graovac and Pisanski $(G P)$ distance number. Graovac and Pisanski [4] established some basic properties of $\delta(G)$ and computed $\delta(G)$ provided $G$ is a path, cube, cycle graph, complete bipartite graph, or lattice graph. Note that the results in this article only hold for the GP distance number and not what is currently referred to in the literature as the Graovac-Pisanski index, namely $\hat{W}(G):=\frac{1}{2}|V(G)|^{2} \delta(G)$.

The GP distance number and the GP index were the subject of prior research by a number of authors. For example, Ashrafi and Shabani [1] computed the GP index of graphs that resulted via standard graph operations on trees. The GP index of truncation graphs, Thorn graphs, and caterpillars were calculated by Iranmanesh and Shabani [7]. Additionally, Knor et al. [8] considered the maximum GP index among all graphs of a fixed order. Note that these results on the GP index have direct implications for the GP distance number.

In this article, we consider a dual problem to that of computing the maximum GP distance number among all graphs of a fixed order; this approach better represents how the GP distance numbers of classes of compounds can predict their physico-chemical properties. Specifically, for a given group $\Gamma$, we establish the possible values of $\delta(G)$ among all graphs $G$ with Aut $G \cong \Gamma$. When Aut $G \cong \Gamma$, we call $G$ a $\Gamma$-graph. Our main result is stated below. 
Theorem 1.1. Given a group $\Gamma$, define

$$
D_{\Gamma}:=\{\delta(G): G \text { is a } \Gamma \text {-graph }\} .
$$

The set $D_{\Gamma}$ is dense in $\left(\inf \left(D_{\Gamma}\right), \infty\right)$. Moreover, for each rational number $q \in\left(\inf \left(D_{\Gamma}\right), \infty\right)$, there exists a $\Gamma$-graph $G$ with $\delta(G)=q$.

Our results will establish the exact value of $\inf \left(D_{\Gamma}\right)$, as well as give two infinite families of $\Gamma$-graphs whose GP distance numbers equal this infimum.

We prove Theorem 1.1 by constructing a family of $\Gamma$-graphs whose vertex orbits under the $\Gamma$-action are not necessarily connected. Consideration of $\Gamma$-graphs whose vertex orbits are all connected yields a more restricted result, Theorem 6.3 , in which the interval of potential GP distance numbers is finite and, moreover, not every rational number in the interval can be obtained as a GP distance number of a graph in the constructed family.

This article is organized as follows. In Section 2, we describe an alternative formula to compute $\delta(G)$ for a given graph $G$, and then use it to state bounds on this invariant in terms of $W(G)$. Next, for a given group $\Gamma$, we construct an infinite family of $\Gamma$-graphs in Section 3. The results of Section 4 establish their associated GP distance numbers, and in Section 5, we present a proof of our main result, Theorem 1.1. Finally, we conclude in Section 6 with a discussion leading to Theorem 6.3 .

\section{Preliminaries}

The definition of $\delta(G)$ for a graph $G$ can be reformulated by considering the orbits of $V(G)$ under the action of Aut $G$. For ease of notation, define

$$
d(v, V):=\sum_{u \in V} d(v, u)
$$

where $v \in V \subseteq V(G)$. Graovac and Pisanski connected this alternative expression for $\delta(G)$ to the Wiener index of the vertex orbits of $G$; we state their results below.

Theorem 2.1 (Graovac and Pisanski [4]). If $V_{0}, V_{1}, \ldots, V_{p-1}$ are the orbits of $V(G)$ determined by Aut $G$ and $v_{i} \in V_{i}$ for each $i \in\{0,1, \ldots, p-1\}$, then

$$
\delta(G)=\frac{1}{|V(G)|} \sum_{i=0}^{p-1} d\left(v_{i}, V_{i}\right)=\frac{2}{|V(G)|} \sum_{i=0}^{p-1} \frac{W\left(V_{i}\right)}{\left|V_{i}\right|} .
$$

For the remainder of this article, we will use Equation (2.1) to compute the GP distance number of a given graph. As simple examples, we calculate the GP distance numbers of both complete graphs and paths below.

Example 2.2. Let $K_{n}$ denote the complete graph with $n$ vertices. If $v \in V\left(K_{n}\right)$, then

$$
\delta\left(K_{n}\right)=\frac{1}{n} d\left(v, V\left(K_{n}\right)\right)=\frac{n-1}{n},
$$

where the first equality holds because $K_{n}$ is vertex-transitive (i.e., $p=1$ ) and the second equality holds because $v$ is adjacent to all vertices in $V\left(K_{n}\right)$ except itself. 
Example 2.3. Let $P_{n}$ denote the path of order $n \geq 2$, and label this graph so that $u_{i} u_{i+1} \in$ $E\left(P_{n}\right)$ for each $i \in\{0,1, \ldots, n-2\}$. Since $P_{n}$ is a $\mathbb{Z}_{2}$-graph, there are $\left\lfloor\frac{n+1}{2}\right\rfloor$ vertex orbits under the action of $\operatorname{Aut}\left(P_{n}\right)$. Set $p=\left\lfloor\frac{n+1}{2}\right\rfloor$ and label these orbits by $V_{0}, V_{1}, \ldots, V_{p-1}$ so that $u_{i} \in V_{i}$ for each $i \in\{0,1, \ldots, p-1\}$. Under these assumptions, $u_{i}$ and $u_{n-1-i}$ comprise the orbit $V_{i}$ and

$$
d\left(u_{i}, V_{i}\right)=d\left(u_{i}, u_{i}\right)+d\left(u_{i}, u_{n-1-i}\right)=0+(n-1-2 i)=n-1-2 i
$$

for all $i \in\{0,1, \ldots, p-1\}$. Therefore,

$$
\delta\left(P_{n}\right)=\frac{1}{n} \sum_{i=0}^{p-1}(\underbrace{n-1-2 i}_{d\left(u_{i}, V_{i}\right)})=\frac{1}{n}\left[p(n-1)-2\left(\frac{1}{2}(p-1) p\right)\right]= \begin{cases}\frac{n}{4} & \text { if } n \text { is even } \\ \frac{n^{2}-1}{4 n} & \text { if } n \text { is odd }\end{cases}
$$

where the first equality holds by Equation (2.1) and the last equality holds because $p=$ $\left\lfloor\frac{n+1}{2}\right\rfloor$.

Paths and complete graphs represent important families of graphs in the context of the Wiener index. In particular, Knor, Škrekovski, and Tepeh [9] observed that if $G$ is a connected graph of order $n$, then

$$
\left(\begin{array}{l}
n \\
2
\end{array}\right)=W\left(K_{n}\right) \leq W(G) \leq W\left(P_{n}\right)=\left(\begin{array}{c}
n+1 \\
3
\end{array}\right) .
$$

For a given graph $G$, this observation allows us to place simple bounds on $\delta(G)$ in terms of $W(G)$.

Lemma 2.4. Let $G$ be a graph. If the induced subgraph on each vertex orbit of $G$ under the action of Aut $G$ is connected with order $k$, then

$$
\frac{k-1}{k} \leq \delta(G) \leq \frac{k^{2}-1}{3 k} .
$$

Proof. Let $V_{0}, V_{1}, \ldots, V_{p-1}$ denote the vertex orbits of $G$ under the action of Aut $G$. Because each orbit has size $k$ and $|V(G)|=k p$, Equation (2.1) implies

$$
\delta(G)=\frac{2}{k^{2} p} \sum_{i=0}^{p-1} W\left(V_{i}\right) .
$$

Combining the equation above with Equation (2.2), we obtain

$$
\frac{k-1}{k}=\frac{2}{k^{2} p} \cdot p\left(\begin{array}{l}
k \\
2
\end{array}\right) \leq \delta(G) \leq \frac{2}{k^{2} p} \cdot p\left(\begin{array}{c}
k+1 \\
3
\end{array}\right)=\frac{k^{2}-1}{3 k},
$$

as desired.

The lower bound stated in Lemma 2.4 is realized by $G=K_{n}$ (see Example 2.2). As demonstrated by Example 2.3, the upper bound in Lemma 2.4 is not realized by $G=P_{n}$. Moreover, we conjecture this upper bound is not sharp under the stated assumptions.

For a given group $\Gamma$, Theorem 1.1 implies that there is no maximum value of $\delta(G)$ among all $\Gamma$-graphs. In fact, the values of GP distance numbers of graphs in general are not bounded; Lemma 2.4 foreshadows how these graphs must be built. To construct a family of graphs with arbitrarily large GP distance numbers, the induced subgraphs on some of the vertex orbits must be disconnected. We continue by constructing such graphs in the next section. 


\section{Graph construction}

To investigate the set $D_{\Gamma}$, we will construct an infinite family of $\Gamma$-graphs, parameterized by non-negative integers $a$ and $c$, from a given $\Gamma$-graph $G$. Specifically, each graph $\varphi_{c}^{a}(G)$ in this family will be constructed by appending to $G$, in a special way, $a$ anti-cliques of order $|V(G)|$ and $c$ cliques of order $|V(G)|$ (see Definition 3.1 below). Every vertex in $\varphi_{c}^{a}(G)$ will have two labels; the superscript of a vertex indicates its distance to $G$ and the subscript label represents the vertex in $G$ it is closest to. The parameters $a$ and $c$ are used in Section 5 to increase and decrease the value of $\delta\left(\varphi_{c}^{a}(G)\right)$, respectively.

Definition 3.1. Let $\Gamma$ be a group, and suppose $G$ is a $\Gamma$-graph with $V(G)=\left\{u_{0}^{0}, u_{1}^{0}, \ldots\right.$, $\left.u_{n-1}^{0}\right\}$. Given $a, c \in \mathbb{N}$, construct a new graph from $G$, denoted $\varphi_{c}^{a}(G)$, with $n(1+a+c)$ vertices and

$$
E(G)+a n+c\left(n+\frac{1}{2} n(n-1)\right)
$$

edges as follows:

1. For each $i \in\{0,1, \ldots, n-1\}$, attach a path of length $a$ to vertex $u_{i}^{0}$ and sequentially label the vertices on that path by $u_{i}^{0}, u_{i}^{1}, u_{i}^{2}, \ldots, u_{i}^{a}$.

2. For each $i \in\{0,1, \ldots, n-1\}$, attach a path of length $c$ to $u_{i}^{0}$ and sequentially label the vertices $w_{i}^{0}, w_{i}^{1}, w_{i}^{2}, \ldots, w_{i}^{c}$, where $w_{i}^{0}:=u_{i}^{0}$; thereupon, provided $c \neq 0$, include the edges $w_{i}^{k} w_{j}^{k}$ for all $k \in\{1,2, \ldots, c\}$ and distinct $i, j \in\{0,1, \ldots, n-1\}$.

Observe that $G$ and $\varphi_{c}^{a}(G)$ are equal when $a=0=c$. The graph $\varphi_{0}^{a}\left(C_{n}\right)$ is depicted in Figure 1, where $C_{n}$ denotes the cycle graph of order $n$. We discuss the structure of the vertex orbits of $\varphi_{c}^{a}(G)$ under the action of Aut $\left(\varphi_{c}^{a}(G)\right)$ in the following remark.

Remark 3.2. Let $\Gamma$ be a group. If $G$ and $\varphi_{c}^{a}(G)$ are both $\Gamma$-graphs, then the vertex orbits of $\varphi_{c}^{a}(G)$ under its $\Gamma$-action depend on the vertex orbits of $G$ under its $\Gamma$-action. In particular, let $V_{0}, V_{1}, \ldots, V_{p-1}$ denote the vertex orbits of $G$ under its $\Gamma$-action. By construction, we obtain $a+c$ vertex orbits of $\varphi_{c}^{a}(G)$ under its $\Gamma$-action for each $V_{i}$, so, in total, $\varphi_{c}^{a}(G)$ has $(1+a+c) p$ vertex orbits under its $\Gamma$-action.

We continue with an example in which we compute the value of $\delta\left(\varphi_{0}^{a}\left(C_{n}\right)\right)$ for all $a, n \in \mathbb{N}$ with $n \geq 3$.

Example 3.3. Let us compute the GP distance number of the graph $\varphi_{0}^{a}\left(C_{n}\right)$, which is illustrated in Figure 1. Recall that $C_{n}$ is vertex-transitive. If $A^{j}$ is the orbit of $u_{0}^{j}$ under the dihedral action of $\operatorname{Aut}\left(\varphi_{0}^{a}\left(C_{n}\right)\right) \cong D_{2 n}$ for all $j \in\{0,1, \ldots, a\}$, then $A^{0}, A^{1}, \ldots, A^{a}$ form a partition of $V\left(\varphi_{0}^{a}\left(C_{n}\right)\right)$. We claim the value of $d\left(u_{0}^{j}, A^{j}\right)$ depends on the parity of $n$.

Consider the vertices $u_{0}^{j}, u_{i}^{j} \in A^{j}$, where $i \in\{1,2, \ldots, n-1\}$ and $j \in\{0,1, \ldots, a\}$. A shortest path between these vertices is constructed by concatenating the $u_{0}^{j}, u_{0}^{0}$-path of length $j$, a $u_{0}^{0}, u_{i}^{0}$-path of minimum length in $C_{n}$, and the $u_{i}^{0}, u_{i}^{j}$-path of length $j$. Therefore, if $n=2 \ell+1$ is odd, then

$$
d\left(u_{0}^{j}, A^{j}\right)=\sum_{i=1}^{n-1} d\left(u_{0}^{j}, u_{i}^{j}\right)=2 \sum_{k=1}^{\ell}(2 j+k)=4 j \ell+\ell(\ell+1)=2(n-1) j+\frac{n^{2}-1}{4},
$$




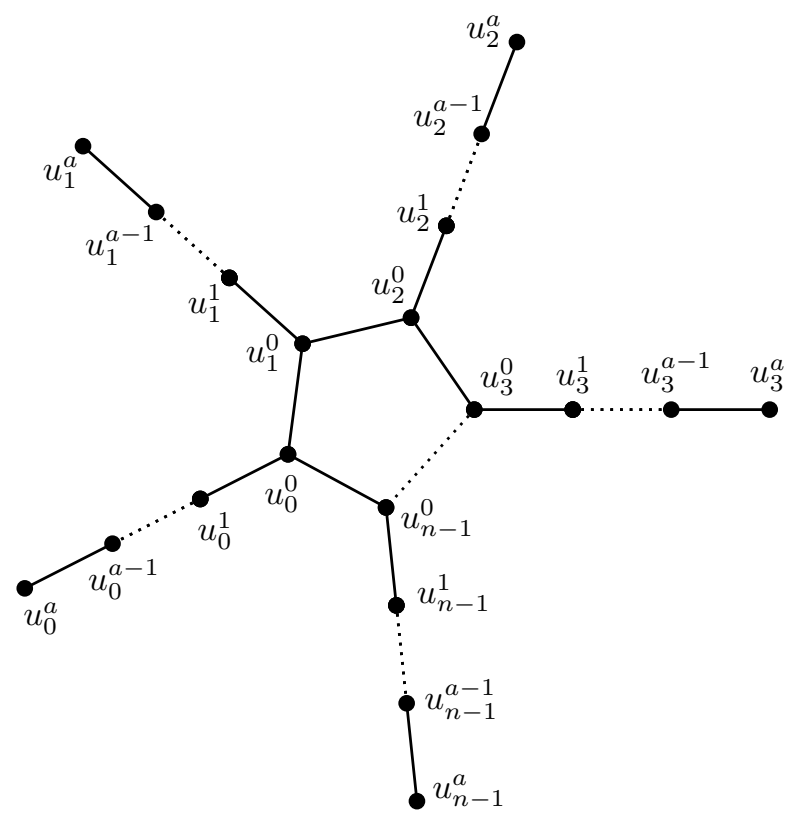

Figure 1: Depiction of the graph $\varphi_{0}^{a}\left(C_{n}\right)$.

and, if $n=2 \ell$ is even, then

$d\left(u_{0}^{j}, A^{j}\right)=\sum_{i=1}^{n-1} d\left(u_{0}^{j}, u_{i}^{j}\right)=(2 j+\ell)+2 \sum_{k=1}^{\ell-1}(2 j+k)=4 j \ell-2 j+\ell^{2}=2(n-1) j+\frac{n^{2}}{4}$.

Since $\left|V\left(\varphi_{0}^{a}\left(C_{n}\right)\right)\right|=n(1+a)$, we have that

$$
\delta\left(\varphi_{0}^{a}\left(C_{n}\right)\right)=\frac{1}{n(1+a)} \sum_{j=0}^{a} d\left(u_{0}^{j}, A^{j}\right)= \begin{cases}\frac{4(n-1) a+n^{2}-1}{4 n} & \text { if } n=2 \ell+1 \\ \frac{4(n-1) a+n^{2}}{4 n} & \text { if } n=2 \ell .\end{cases}
$$

The statements in Remark 3.2 are based on the assumption that $G$ and $\varphi_{c}^{a}(G)$ have isomorphic automorphism groups. The following proposition proves that this is almost always the case.

Proposition 3.4. Let $\Gamma$ be a group. If $G$ is a nontrivial connected $\Gamma$-graph and either $a \neq 0$ or $G$ is not a complete graph, then $\varphi_{c}^{a}(G)$ is also a $\Gamma$-graph.

Proof. To prove that $\Gamma$ is isomorphic to a subgroup of Aut $\left(\varphi_{c}^{a}(G)\right)$, we note that each element of Aut $G$ induces a (subscript) label-preserving automorphism of $\varphi_{c}^{a}(G)$. In particular, if $\sigma \in \operatorname{Aut} G$, then $\sigma$ induces a permutation on $\{0,1, \ldots, n-1\}$, denoted $\rho_{\sigma}$, such that $\rho_{\sigma}(i)$ is the subscript of $\sigma\left(u_{i}^{0}\right)$ for all $i \in\{0,1, \ldots, n-1\}$. Define the $\operatorname{map} \pi_{\sigma}: V\left(\varphi_{c}^{a}(G)\right) \rightarrow V\left(\varphi_{c}^{a}(G)\right)$ by $\pi_{\sigma}\left(u_{i}^{j}\right)=u_{\rho_{\sigma}(i)}^{j}$ and $\pi_{\sigma}\left(w_{i}^{k}\right)=w_{\rho_{\sigma}(i)}^{k}$ for all $j \in\{0,1, \ldots, a\}$ and $k \in\{0,1, \ldots, c\}$. Since $\pi_{\sigma}$ preserves the adjacency relations in $\varphi_{c}^{a}(G)$ and $\Gamma \cong\left\{\pi_{\sigma}: \sigma \in \operatorname{Aut} G\right\}, \Gamma$ is isomorphic to a subgroup of Aut $\left(\varphi_{c}^{a}(G)\right)$. 
It remains to prove that any element of Aut $\left(\varphi_{c}^{a}(G)\right)$ is equal to $\pi_{\sigma}$ for some $\sigma \in$ Aut $G$. Clearly if $a=0=c$, then $\varphi_{c}^{a}(G)=G$ and the proposition holds. Thus, in what follows we assume that at least one of $a$ or $c$ is nonzero.

Suppose $a \neq 0$, and consider the image of the degree-1 vertex $u_{i}^{a}$ under $\psi \in$ Aut $\left(\varphi_{c}^{a}(G)\right)$, where $i \in\{0,1, \ldots, n-1\}$. Since the only vertices in $\varphi_{c}^{a}(G)$ that have degree 1 are of the form $u_{\ell}^{a}$, it follows that $\psi\left(u_{i}^{a}\right)=u_{\ell}^{a}$ for some $\ell \in\{0,1, \ldots, n-1\}$. In turn, $\psi\left(u_{i}^{a-1}\right)=u_{\ell}^{a-1}$ because $u_{i}^{a-1}$ and $u_{\ell}^{a-1}$ are the only neighbors of $u_{i}^{a}$ and $u_{\ell}^{a}$ in $\varphi_{c}^{a}(G)$, respectively. Proceeding by induction, assume that $\psi\left(u_{i}^{j^{\prime}}\right)=u_{\ell}^{j^{\prime}}$ for all $j^{\prime} \in\{j, j+1, \ldots, a\}$. If $j \geq 1$, then $u_{i}^{j}$ has exactly two neighbors, namely $u_{i}^{j+1}$ and $u_{i}^{j-1}$, while $u_{\ell}^{j+1}$ and $u_{\ell}^{j-1}$ are the only neighbors of vertex $u_{\ell}^{j}$. In this case, $\psi\left(u_{i}^{j-1}\right)=u_{\ell}^{j-1}$ as $\psi\left(u_{i}^{j+1}\right)=u_{\ell}^{j+1}$ by induction. Therefore, $\psi\left(u_{i}^{j}\right)=u_{\ell}^{j}$ for all $j \in\{0,1, \ldots, a\}$.

Now define $W^{k}:=\left\{w_{0}^{k}, w_{1}^{k}, \ldots, w_{n-1}^{k}\right\}$ for each $k \in\{0,1, \ldots, c\}$. If $c \neq 0$, then each vertex in $W^{c}$ has degree $n$, and thus $\psi\left(w_{i}^{c}\right)$ is also a vertex of degree $n$ in $\varphi_{c}^{a}(G)$. The only vertices in $\varphi_{c}^{a}(G)$ that have degree $n$ are in $W^{0} \cup W^{c}$. However, each element in $W^{c}$ is adjacent to at least $n-1$ vertices of degree $n$, and because $G$ is not a complete graph or $a \neq 0$, each vertex in $W^{0}=V(G)$ is adjacent to at most $n-2$ vertices of degree $n$. Consequently, $W^{c}$ is $\psi$-invariant; assume that $\psi\left(w_{i}^{c}\right)=w_{m}^{c}$ for some $m \in$ $\{0,1, \ldots, n-1\}$. Both $w_{i}^{c}$ and $w_{m}^{c}$ have exactly one neighbor that is not an element of $W^{c}$; hence, $\psi\left(w_{i}^{c-1}\right)=w_{m}^{c-1}$ and we claim that $\psi\left(w_{i}^{k}\right)=w_{m}^{k}$ for all $k \in\{0,1, \ldots, c\}$. Since this claim holds for $k \in\{c-1, c\}$, we again proceed by induction. Assume that $\psi\left(w_{i}^{k^{\prime}}\right)=w_{m}^{k^{\prime}}$ for all $k^{\prime} \in\{k, k+1, \ldots, c\}$. When $k \geq 1$, the only neighbors of $w_{i}^{k}$ not in $W^{k}$ are $w_{i}^{k+1}$ and $w_{i}^{k-1}$; moreover, $w_{m}^{k+1}$ and $w_{m}^{k-1}$ are the only neighbors of $w_{m}^{k}$ not in $W^{k}$. Since $\psi\left(w_{i}^{k+1}\right)=w_{m}^{k+1}$ by induction, it follows that $\psi\left(w_{i}^{k-1}\right)=w_{m}^{k-1}$ and the claim holds.

Our work above proves that $\psi\left(u_{i}^{j}\right)=u_{\ell}^{j}$ for all $j \in\{0,1, \ldots, a\}$ and that $\psi\left(w_{i}^{k}\right)=$ $w_{m}^{k}$ for all $k \in\{0,1, \ldots, c\}$. Since $u_{i}^{0}=w_{i}^{0}$ by definition of $\varphi_{c}^{a}(G)$, we have $\ell=$ $m$. Consequently, there exists $\sigma \in \operatorname{Aut}(G)$ such that $\psi=\pi_{\sigma}$, and $\varphi_{c}^{a}(G)$ is also a $\Gamma$ graph.

We are now ready to compute the GP distance number of $\varphi_{c}^{a}(G)$ when the graphs $G$ and $\varphi_{c}^{a}(G)$ have isomorphic automorphism groups.

\section{GP distance number of $\varphi_{c}^{a}(G)$}

If $G$ and $\varphi_{c}^{a}(G)$ have isomorphic automorphism groups, then the value of $\delta\left(\varphi_{c}^{a}(G)\right)$ naturally depends on the value $\delta(G)$; however, it also depends on the value of $c$ in a special way. In particular, if $c \neq 0$, then the distance between any two vertices of $G$ is at most 3 . Recalling that $V_{0}, V_{1}, \ldots, V_{p-1}$ are the vertex orbits of $G$ under the action of Aut $G$, we define

$$
\delta^{\prime}(c, G):= \begin{cases}\delta(G) & \text { if } c=0 \\ \delta_{3}(G) & \text { if } c \neq 0\end{cases}
$$

where

$$
\delta_{3}(G):=\frac{1}{|V(G)|} \sum_{i=0}^{p-1} d_{3}\left(u_{i}, V_{i}\right) \quad \text { and } \quad d_{3}\left(u_{i}, V_{i}\right):=\sum_{u \in V_{i}} \min \left\{d\left(u_{i}, u\right), 3\right\} .
$$

With this notation in hand, we compute the value of $\delta\left(\varphi_{c}^{a}(G)\right)$ below. 
Proposition 4.1. Let $\Gamma$ be a group, and assume that $G$ and $\varphi_{c}^{a}(G)$ are both $\Gamma$-graphs. If $G$ has order $n$ and $p$ vertex orbits under the action of Aut $G$, then

$$
\delta\left(\varphi_{c}^{a}(G)\right)=\frac{(n-p)\left(a^{2}+a+c\right)+n(a+1) \delta^{\prime}(c, G)}{n(1+a+c)} .
$$

Proof. Let $V_{0}, V_{1}, \ldots, V_{p-1}$ denote the $p$ vertex orbits of $G$ under the action of Aut $G$. After a possible relabelling of $V(G)$, assume that $u_{i}^{0} \in V_{i}$ for all $i \in\{0,1, \ldots, p-1\}$. For each $V_{i}$, there are $a+c$ associated vertex orbits of $\varphi_{c}^{a}(G)$ under the action of $\operatorname{Aut}\left(\varphi_{c}^{a}(G)\right)$ by Remark 3.2; label these orbits by $A_{i}^{1}, A_{i}^{2}, \ldots, A_{i}^{a}$ and $C_{i}^{1}, C_{i}^{2}, \ldots, C_{i}^{c}$, where $u_{i}^{j} \in A_{i}^{j}$ for $j \in\{1,2, \ldots, a\}$ and $w_{i}^{k} \in C_{i}^{k}$ for $k \in\{1,2, \ldots, c\}$. Under these assumptions

$$
\delta\left(\varphi_{c}^{a}(G)\right)=\frac{1}{\left|V\left(\varphi_{c}^{a}(G)\right)\right|}\left(\sum_{j=0}^{a} \sum_{i=0}^{p-1} d\left(u_{i}^{j}, A_{i}^{j}\right)+\sum_{k=1}^{c} \sum_{i=0}^{p-1} d\left(w_{i}^{k}, C_{i}^{k}\right)\right),
$$

where $A_{i}^{0}=V_{i}$ for $i \in\{0,1, \ldots, p-1\}$. We evaluate each of these sums in one of the following cases.

First, observe that $d\left(w_{i}^{k}, C_{i}^{k}\right)=\left|C_{i}^{k}\right|-1$ for all $k \in\{1,2, \ldots, c\}$ as the induced subgraph on $C_{i}^{k}$ is a clique. Since

$$
\sum_{k=1}^{c}\left|C_{i}^{k}\right|=c\left|V_{i}\right| \quad \text { and } \quad \sum_{i=0}^{p-1}\left|V_{i}\right|=|V(G)|=n,
$$

it follows that

$$
\sum_{k=1}^{c} \sum_{i=0}^{p-1} d\left(w_{i}^{k}, C_{i}^{k}\right)=\sum_{i=0}^{p-1} \sum_{k=1}^{c}\left(\left|C_{i}^{k}\right|-1\right)=\sum_{i=0}^{p-1} c\left(\left|V_{i}\right|-1\right)=c(n-p) .
$$

For the second case, if $u_{\ell}^{0} \in A_{i}^{0}$, then a shortest path between vertices $u_{i}^{j} \in A_{i}^{j}$ and $u_{\ell}^{j} \in A_{i}^{j}$ is constructed by concatenating the following three paths:

1. the $u_{i}^{j}, u_{i}^{0}$-path in $\varphi_{c}^{a}(G)$ of length $j$;

2. a $u_{i}^{0}, u_{\ell}^{0}$-path of minimum length in $G$ if $c=0$ or in $\varphi_{1}^{0}(G)$ provided $c \neq 0$; and

3. the $u_{\ell}^{0}, u_{\ell}^{j}$-path in $\varphi_{c}^{a}(G)$ of length $j$.

It follows that

$$
d\left(u_{i}^{j}, A_{i}^{j}\right)=2 j\left(\left|A_{i}^{j}\right|-1\right)+d^{\prime}\left(c, u_{i}^{0}, A_{i}^{0}\right),
$$

where

$$
d^{\prime}\left(c, u_{i}^{0}, A_{i}^{0}\right):= \begin{cases}d\left(u_{i}^{0}, A_{i}^{0}\right) & \text { if } c=0 \\ d_{3}\left(u_{i}^{0}, A_{i}^{0}\right) & \text { if } c \neq 0 .\end{cases}
$$

Since $\left|A_{i}^{j}\right|=\left|V_{i}\right|$ for all $j \in\{0,1, \ldots, a\}$, we have

$$
\begin{aligned}
\sum_{j=0}^{a} \sum_{i=0}^{p-1} d\left(u_{i}^{j}, A_{i}^{j}\right) & =\sum_{i=0}^{p-1} \sum_{j=0}^{a}(\underbrace{2 j\left(\left|A_{i}^{j}\right|-1\right)+d^{\prime}\left(c, u_{i}^{0}, A_{i}^{0}\right)}_{d\left(u_{i}^{j}, A_{i}^{j}\right)}) \\
& =\sum_{i=0}^{p-1}\left(2 \frac{1}{2} a(a+1)\left(\left|V_{i}\right|-1\right)+(a+1) d^{\prime}\left(c, u_{i}^{0}, A_{i}^{0}\right)\right) \\
& =a(a+1)(n-p)+n(a+1) \delta^{\prime}(c, G) .
\end{aligned}
$$


Since $\left|V\left(\varphi_{c}^{a}(G)\right)\right|=n(1+a+c)$, combining Equations (4.2) and (4.3) with Equation (4.1) yields

$$
\delta\left(\varphi_{c}^{a}(G)\right)=\frac{(n-p)\left(a^{2}+a+c\right)+n(a+1) \delta^{\prime}(c, G)}{n(1+a+c)},
$$

as desired.

Consider the value of $\delta\left(\varphi_{c}^{a}(G)\right)$ given in Proposition 4.1 for a fixed graph $G$. The parameters $a$ and $c$ can be used to increase and decrease the value of $\delta\left(\varphi_{c}^{a}(G)\right)$, respectively; that is,

$$
\lim _{a \rightarrow \infty} \delta\left(\varphi_{c}^{a}(G)\right)=\infty \quad \text { and } \quad \lim _{c \rightarrow \infty} \delta\left(\varphi_{c}^{a}(G)\right)=\frac{n-p}{n},
$$

provided $c$ and $a$ are fixed, respectively. There are several infinite families of order- $n$ graphs whose GP distance numbers are equal to $\frac{n-p}{n}$, where $p$ is the number of vertex orbits under the action of their respective automorphism groups. These families arise when the induced subgraph on every vertex orbit is a clique; Example 2.2 demonstrates that the complete graphs $K_{n}$ comprise one such family. The following example establishes a second such family of graphs that, in contrast, are not vertex-transitive under the action of their respective automorphism groups.

Example 4.2. Let $\mathbb{Z}_{k}$ denote the cyclic group of order $k$, where $k \geq 3$. In this example, we construct an infinite family of $\mathbb{Z}_{k}$-graphs, denoted by $G_{n}$; each graph $G_{n}$ has order $n=6 k$ and $p=6$ edge orbits under the action of $\operatorname{Aut}\left(G_{n}\right)$. We will prove that $\delta\left(G_{n}\right)=\frac{n-p}{n}$.

Define the order- 7 gadget graph $H$ with edge set

$$
E(H)=\left\{h_{0} h_{1}, h_{1} h_{2}, h_{1} h_{4}, h_{2} h_{3}, h_{2} h_{5}, h_{5} h_{6}\right\},
$$

which is depicted in Figure 2(A). Let $C_{k}$ denote the cycle graph of order $k$, and label its edges so that $v_{i} v_{i+1} \in E\left(C_{k}\right)$ for all $i \in\{0,1, \ldots, k-2\}$. Replace each edge in $C_{k}$ with a copy of $H$, where the vertices $v_{i}$ and $v_{i+1}$ are identified with $h_{0}$ and $h_{3}$, respectively; we call the resulting graph $H(k)$. The graph $H(4)$ is illustrated in Figure 2(B). Observe that $H(k)$ is a $\mathbb{Z}_{k}$-graph with order $n=6 k$, which has six size- $k$ vertex orbits under the action of Aut $(H(k))$.

Finally, we construct the graph $G_{n}$ by including the $3(k-1) k$ edges necessary to turn each vertex orbit of $H(k)$ into a clique. By design $G_{n}$ is also a $\mathbb{Z}_{k}$-graph, where each of its six edge orbits under the action of $\operatorname{Aut}\left(G_{n}\right)$ is a clique of order $k$. Its GP distance number is

$$
\delta\left(G_{n}\right)=\frac{n-6}{n}=\frac{k-1}{k},
$$

as desired.

\section{Proof of Theorem 1.1}

In this section, we will prove our main result, Theorem 1.1. To do so, we make use of the following proposition.

Proposition 5.1. Let $\Gamma$ be a group, and suppose $G$ is a nontrivial connected $\Gamma$-graph with order $n$ and $p$ vertex orbits under the action of Aut $G$. For any rational number $q \in\left(\frac{n-p}{n}, \infty\right)$, there exist $a, c \in \mathbb{N}$ such that $\delta\left(\varphi_{c}^{a}(G)\right)=q$. 


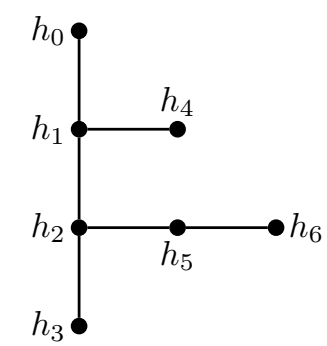

(a) The gadget graph $H$

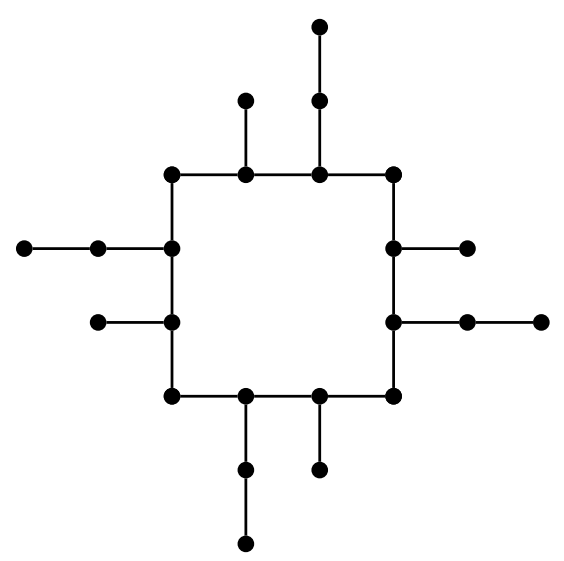

(b) The $\mathbb{Z}_{4}$-graph $H(4)$

Figure 2: Depictions of the graphs $H$ and $H(4)$, which were defined in Example 4.2.

Proof. Choose $r, s \in \mathbb{N}$ such that $q=\frac{r}{s}$, and define

$$
b:=2 \max \left\{1,\left\lceil\frac{n r-n s \delta_{3}(G)}{(n-p) s}\right\rceil\right\} .
$$

Let

$$
a:=(n r-(n-p) s) b-1,
$$

and notice that $a \geq 0$ because $\frac{n-p}{n}<q=\frac{r}{s}$. Now define

$$
c:=-\left(n r-(n-p) a s-n s \delta_{3}(G)\right) b .
$$

Since $G$ has order $n, n \delta_{3}(G)$ is an integer, and thus $c$ is as well. In fact, $c \in \mathbb{N}$ because the inequality

$$
a=(n r-(n-p) s) b-1 \geq b-1 \geq \frac{1}{2} b \geq \frac{n r-n s \delta_{3}(G)}{(n-p) s}
$$

implies that

$$
n r-(n-p) a s-n s \delta_{3}(G)
$$

is nonpositive. Consequently, our choices of $a$ and $c$ are valid when considering the graph $\varphi_{c}^{a}(G)$, and since $a \neq 0, \varphi_{c}^{a}(G)$ is also a $\Gamma$-graph by Proposition 3.4. Proposition 4.1 then implies that the GP distance number of $\varphi_{c}^{a}(G)$ is

$$
\delta\left(\varphi_{c}^{a}(G)\right)=\frac{(n-p)\left(a^{2}+a+c\right)+n(a+1) \delta^{\prime}(c, G)}{n(1+a+c)} .
$$

A tedious algebraic computation shows that combining our choices of $a$ and $c$ (stated in Equations (5.1) and (5.2)) with the equation above yields $\delta\left(\varphi_{c}^{a}(G)\right)=\frac{r}{s}$, as desired.

The equation $\delta\left(\varphi_{c}^{a}(G)\right)=q$ that appears in Proposition 5.1 does not have a unique solution. In fact, taking any integer value of $b$ greater than the one specified in the proof will also yield a choice of $a$ and $c$ which satisfies the theorem. We now provide an example showing that it is also possible to obtain smaller values of $a$ and $c$ which work. 
Example 5.2. Let $G$ be $K_{4}-e$ for any edge $e$ of $K_{4}$, in which case Aut $G \cong \mathbb{Z}_{2} \times \mathbb{Z}_{2}$ and $\delta(G)=\frac{3}{4}$. Applying the proof of Proposition 5.1 with $q=\frac{4}{5}$, we obtain $b=2$ and then $a=11$ and $c=218$. However, we can in fact take $b=1$ and still obtain a solution to $\delta\left(\varphi_{c}^{a}(G)\right)=q$, namely $a=5$ and $c=49$. The solution with the smallest possible values of both $a$ and $c$, not obtainable through the construction in that proof, is $a=1$ and $c=3$.

We conclude this section with a proof of our main result.

Proof of Theorem 1.1. Let the group $\Gamma$ be given, and recall that

$$
D_{\Gamma}:=\{\delta(G): G \text { is a } \Gamma \text {-graph }\} .
$$

Frucht [3] proved that there exists a graph whose automorphism group is isomorphic to $\Gamma$; among all such $\Gamma$-graphs $G$ with order $n_{G}$ and with $p_{G}$ vertex orbits under the action of Aut $G$, choose $G$ so that $\frac{n_{G}-p_{G}}{n_{G}}$ is minimal. Under these assumptions, if $G$ has order $n$ and $p$ vertex orbits under the action of Aut $G$, then

$$
\inf \left(D_{\Gamma}\right)=\frac{n-p}{n} .
$$

For each rational number $q \in\left(\inf \left(D_{\Gamma}\right), \infty\right)$, there exists a $\Gamma$-graph with GP distance number equal to $q$ by Proposition 5.1. Consequently, $D_{\Gamma}$ is dense in $\left(\inf \left(D_{\Gamma}\right), \infty\right)$, as the rational numbers are dense in this interval. The result now follows.

\section{Graphs with connected vertex orbits}

For a given group $\Gamma$, Theorem 1.1 proved that there was no maximum value of $\delta(G)$ among all $\Gamma$-graphs; such arbitrarily large values of $\delta(G)$ were obtained from graphs with disconnected induced subgraphs on the vertex orbits of $G$ under the action of Aut $G$. If we assume that the induced subgraph on every vertex orbit of $G$ under the action of Aut $G$ is connected, then we obtain a bounded interval of potential GP distance numbers. While these stricter assumptions preserve density, we no longer can produce a graph with a given GP distance number using a similar construction. We will conclude this article with a result analogous to that of Theorem 1.1 which makes the aforementioned connectedness assumption.

Let the group $\Gamma$ be given. If a $\Gamma$-set $V$ has size $n$, let $G_{\Gamma, n}$ denote any choice of a connected graph on the $\Gamma$-set $V$ which has a $\Gamma$-action compatible with the $\Gamma$-action on $V$ and has the maximum possible GP distance number among all such graphs. Note that $G_{\Gamma, n}$ need not be a $\Gamma$-graph. We use $\delta_{\Gamma}\left(G_{\Gamma, n}\right)$ to denote the GP distance number obtained by considering the $\Gamma$-action on $G_{\Gamma, n}$ rather than the action of $\operatorname{Aut}\left(G_{\Gamma, n}\right)$.

Suppose now that $G$ is a $\Gamma$-graph with $p$ orbits $V_{0}, V_{1}, \ldots, V_{p-1}$ of sizes $n_{0}, n_{1}, \ldots$, $n_{p-1}$, respectively. Each orbit itself has a $\Gamma$-action, so we consider the graphs $G_{\Gamma, n_{0}}, \ldots$, $G_{\Gamma, n_{p-1}}$; let $\widehat{G}_{\Gamma}$ denote $G_{\Gamma, n_{0}} \sqcup \cdots \sqcup G_{\Gamma, n_{p-1}}$, where $\sqcup$ denotes disjoint union. Define

$$
\hat{\delta}(G):=\frac{1}{n_{0}+\cdots+n_{p-1}} \sum_{i=0}^{p-1} n_{i} \delta_{\Gamma}\left(G_{\Gamma, n_{i}}\right) ;
$$

$\hat{\delta}(G)$ is the maximum possible GP distance number relative to $\Gamma$ for all graphs with a $\Gamma$-action and vertex set the $\Gamma$-set $V(G)$. Note, however, that $\operatorname{Aut}\left(\widehat{G}_{\Gamma}\right)$ may contain an isomorphic copy of $\Gamma$ as a proper subgroup. 
Definition 6.1. Let $\Gamma$ be a group, and suppose $G$ is a $\Gamma$-graph with vertices $u_{0}^{0}, u_{1}^{0}, \ldots, u_{n-1}^{0}$ and vertex orbits $V_{0}, V_{1}, \ldots, V_{p-1}$. Without loss of generality, we assume that $u_{i}^{0} \in V_{i}$ for each $i \in\{0,1, \ldots, p-1\}$. We define a new graph $\hat{\varphi}_{c}^{a}(G)$ iteratively with respect to the natural numbers $c$ and $a$ as follows. Given $\hat{\varphi}_{c}^{a}(G)$, define $\hat{\varphi}_{c}^{a+1}(G)$ to be the graph obtained by carrying out the following steps:

1. introduce new vertices $u_{0}^{a+1}, u_{1}^{a+1}, \ldots, u_{n-1}^{a+1}$; we refer to these vertices as being in "level $a+1 " ;$

2. connect these new vertices with new edges $u_{i}^{a} u_{i}^{a+1}$ for each $i \in\{0,1, \ldots, n-1\}$; and

3. for each orbit $V_{i}$, add new edges to build a copy of the $\Gamma$-graph $G_{\Gamma,\left|V_{i}\right|}$ on the orbit of vertices in level $a+1$ corresponding to the $\Gamma$-set $V_{i}$.

Given $\hat{\varphi}_{c}^{a}(G)$, let $w_{i}^{0}:=u_{i}^{0}$ for each $i \in\{0,1, \ldots, n-1\}$. Define $\hat{\varphi}_{c+1}^{a}(G)$ by connecting an $n$-clique on new vertices $w_{i}^{c+1}$ with new edges $w_{i}^{c} w_{i}^{c+1}$ for each $i \in\{0,1, \ldots, n-1\}$.

Note that, under the $\Gamma$-action, we have enhanced $G$ with $c p$ orbits whose induced subgraphs are cliques and with ap orbits whose induced subgraphs are disjoint unions of connected GP-distance-number-maximizing graphs.

Let $G$ be a $\Gamma$-graph for a given group $\Gamma$. The following proposition shows that $\hat{\varphi}_{c}^{a}(G)$ is also a $\Gamma$-graph in most cases. We omit its proof, which is similar to the proof of Proposition 3.4 .

Proposition 6.2. Let $\Gamma$ be a group, and suppose $G$ is a nontrivial connected $\Gamma$-graph that is not complete. If either $c \neq 0$ or $G \neq \widehat{G}_{\Gamma}$, then $\hat{\varphi}_{c}^{a}(G)$ is also a $\Gamma$-graph.

We now present our result analogous to Theorem 1.1 that makes an assumption on the connectedness of graphs.

Theorem 6.3. Let $\Gamma$ be a group. If $G$ is a connected $\Gamma$-graph of order $n$ having $p$ vertex orbits, each of which induces a connected subgraph of $G$, then

$$
\left\{\delta\left(\hat{\varphi}_{c}^{a}(G)\right) \mid a, c \in \mathbb{N} \text { and } \hat{\varphi}_{c}^{a}(G) \text { is a } \Gamma \text {-graph }\right\}
$$

is dense in $\left(\frac{n-p}{n}, \hat{\delta}(G)\right)$.

Proof. Given any $\epsilon>0$ and any $q \in\left(\frac{n-p}{n}, \hat{\delta}(G)\right)$, it suffices to find $a^{\prime}, c^{\prime} \in \mathbb{N}$ such that $\left|q-\delta\left(\hat{\varphi}_{c^{\prime}}^{a^{\prime}}(G)\right)\right|<\epsilon$. We first determine an expression for $\delta\left(\hat{\varphi}_{c}^{a}(G)\right)$, and then explain how to choose $a^{\prime}$ and $c^{\prime}$.

Let $V_{0}, V_{1}, \ldots, V_{p-1}$ be the $\Gamma$-orbits in $V(G)$. For each $V_{i}$, there are $a+c$ associated vertex orbits of $\hat{\varphi}_{c}^{a}(G)$ under the action of $\operatorname{Aut}\left(\hat{\varphi}_{c}^{a}(G)\right)$; for $i \in\{0,1, \ldots, p-1\}$, label these orbits by $A_{i}^{1}, A_{i}^{2}, \ldots, A_{i}^{a}$ and $C_{i}^{1}, C_{i}^{2}, \ldots, C_{i}^{c}$, where $u_{i}^{j} \in A_{i}^{j}$ for $j \in\{1,2, \ldots, a\}$ and $w_{i}^{k} \in C_{i}^{k}$ for $k \in\{1,2, \ldots, c\}$. For $X \in\left\{G, \widehat{G}_{\Gamma}\right\}$, let $d_{X}$ denote the distance function in $X$, and let $d_{X, 3}$ denote the function given by $\min \left(d_{X}(u, v), 3\right)$ for vertices $u, v \in V(X)$. Write $d_{G}^{\prime}=d_{G}$ for $c=0$ and $d_{G}^{\prime}=d_{G, 3}$ for $c \geq 1$.

For each $i \in\{0,1, \ldots, p-1\}$ and any $k$, any two distinct vertices in $C_{i}^{k}$ are at distance 1 from each other. Choosing a representative in each orbit $C_{1}^{k}, C_{2}^{k}, \ldots, C_{p-1}^{k}$, we find that the total distance over all the orbits in level $k$ is

$$
\sum_{i=0}^{p-1}\left(\left|C_{i}^{k}\right|-1\right)=n-p
$$


For $i \in\{0,1, \ldots, p-1\}$ and any $j$, a shortest path between any two vertices $u_{\ell}^{j}, u_{m}^{j}$ in $A_{i}^{j}$ is either a shortest path in layer $j$, or is a path obtained by concatenating a shortest $u_{\ell}^{j}, u_{\ell}^{0}$-path and a shortest $u_{m}^{0}, u_{m}^{j}$-path with a shortest $u_{\ell}^{0}, u_{m}^{0}$-path in $G$ if $c=0$ and with a shortest $u_{\ell}^{0}, u_{m}^{0}$-path in $\hat{\varphi}_{1}^{0}(G)$ if $c>0$. Thus, the length of a shortest $u_{\ell}^{j}, u_{m}^{j}$-path is

$$
\min \left\{d_{\widehat{G}_{\Gamma}}\left(u_{\ell}^{j}, u_{m}^{j}\right), 2 j+d_{G}^{\prime}\left(u_{\ell}^{0}, u_{m}^{0}\right)\right\} .
$$

Writing $\operatorname{diam}(X)$ for the length of a longest path in graph $X$, if $j \geq \operatorname{diam}\left(\widehat{G}_{\Gamma}\right) / 2$ then we have

$$
\min \left\{d_{\widehat{G}_{\Gamma}}\left(u_{\ell}^{0}, u_{m}^{0}\right), 2 j+d_{G}^{\prime}\left(u_{\ell}^{0}, u_{m}^{0}\right)\right\}=d_{\widehat{G}_{\Gamma}}\left(u_{\ell}^{0}, u_{m}^{0}\right) .
$$

Note that, to prove the result, it suffices to presume that $a>\operatorname{diam}\left(\widehat{G}_{\Gamma}\right) / 2$. Choosing a representative in each orbit, we can calculate the total distance for levels 0 to $\left\lceil\operatorname{diam}\left(\widehat{G}_{\Gamma}\right) / 2\right\rceil$; write $D$ for this value. Also, for each $j>\left\lceil\operatorname{diam}\left(\widehat{G}_{\Gamma}\right) / 2\right\rceil$, the total distance in level $j$ is $n \hat{\delta}(G)$. Thus, we have

$$
\delta\left(\hat{\varphi}_{c}^{a}(G)\right)=\frac{(n-p) c+D+\left(a-\left\lceil\operatorname{diam}\left(\widehat{G}_{\Gamma}\right) / 2\right\rceil\right) n \hat{\delta}(G)}{(1+a+c) n} .
$$

In order to choose appropriate $a$ and $c$, observe first that, for any positive $a, c \in \mathbb{N}$, we have

$$
\begin{aligned}
\delta\left(\hat{\varphi}_{c-1}^{a}(G)\right)-\delta\left(\hat{\varphi}_{c}^{a}(G)\right) & =\frac{D+\left(a-\left\lceil\operatorname{diam}\left(\widehat{G}_{\Gamma}\right) / 2\right\rceil\right) n \hat{\delta}(G)-(n-p)(a+1)}{(a+c)(1+a+c) n} \\
& <\frac{D+a n \hat{\delta}(G)}{(a+c)^{2} n} .
\end{aligned}
$$

Let $\Delta(a, c)$ denote this upper bound, and note that $\Delta(a, c)$ has negative derivative with respect to both $a$ and to $c$.

We now choose $a^{\prime}$ and $c^{\prime}$. Since

$$
\lim _{a \rightarrow \infty} \delta\left(\hat{\varphi}_{0}^{a}(G)\right)=\hat{\delta}(G)>q
$$

we can choose $a^{\prime} \in \mathbb{N}$ so that $a^{\prime}>\left\lceil\operatorname{diam}\left(\widehat{G}_{\Gamma}\right) / 2\right\rceil, \Delta\left(a^{\prime}, 0\right)<\epsilon$, and $\delta\left(\hat{\varphi}_{0}^{a^{\prime}}(G)\right)>q$. Because

$$
\lim _{c \rightarrow \infty} \delta\left(\hat{\varphi}_{c}^{a^{\prime}}(G)\right)=\frac{n-p}{n}<q
$$

we can then choose

$$
c^{\prime}:=\min \left\{c \in \mathbb{N} \mid \delta\left(\hat{\varphi}_{c}^{a^{\prime}}(G)\right) \leq q\right\} .
$$

Observe that $c^{\prime}>0$ because we have chosen $a^{\prime}$ to ensure that $\delta\left(\hat{\varphi}_{0}^{a^{\prime}}(G)\right)>q$. Since

$$
\delta\left(\hat{\varphi}_{c^{\prime}}^{a^{\prime}}(G)\right)<q \leq \delta\left(\hat{\varphi}_{c^{\prime}-1}^{a^{\prime}}(G)\right)
$$

we have

$$
q-\delta\left(\hat{\varphi}_{c^{\prime}}^{a^{\prime}}(G)\right)<\Delta\left(a^{\prime}, c^{\prime}\right)<\Delta\left(a^{\prime}, 0\right)<\epsilon,
$$

as desired. Furthermore, since $c^{\prime}>0$, Proposition 6.2 guarantees that $\hat{\varphi}_{c^{\prime}}^{a^{\prime}}(G)$ is a $\Gamma$ graph. 
Let $\Gamma$ be a group, and suppose $G$ is a connected $\Gamma$-graph of order $n$ with $p$ vertex orbits under the action of Aut $G$. If the induced subgraph on each vertex orbit of $G$ is connected, then we claim that there exists infinitely many rational numbers in $\left(\frac{n-p}{n}, \hat{\delta}(G)\right)$ that are not the GP distance numbers of graphs of the form $\hat{\varphi}_{c}^{a}(G)$. We demonstrate our claim with the following example.

Example 6.4. Let $G$ be the graph constructed from an 8-cycle on vertices $u_{0}^{0}, u_{1}^{0}, u_{2}^{0}, \ldots, u_{7}^{0}$ and a 4-cycle on vertices $u_{8}^{0}, u_{9}^{0}, u_{10}^{0}, u_{11}^{0}$, by including edges

$$
u_{0}^{0} u_{8}^{0}, u_{1}^{0} u_{8}^{0}, u_{2}^{0} u_{9}^{0}, u_{3}^{0} u_{9}^{0}, u_{4}^{0} u_{10}^{0}, u_{5}^{0} u_{10}^{0}, u_{6}^{0} u_{11}^{0} \text {, and } u_{7}^{0} u_{11}^{0} \text {. }
$$

The graph $G$, which is illustrated in Figure 3, is a $D_{8}$-graph with two vertex orbits under the action of Aut $G$ (here $D_{8}$ denotes the dihedral group of order 8).

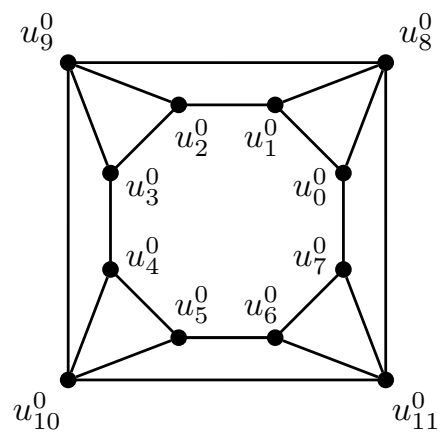

Figure 3: The $D_{8}$-graph $G$ constructed in Example 6.4.

Observe that $\widehat{G}_{D_{8}}$ is equal to $C_{8} \sqcup C_{4}$. Moreover, $\delta(G)=\frac{20}{12}=\hat{\delta}(G)$, and thus Theorem 6.3 established that $\left\{\delta\left(\hat{\varphi}_{c}^{a}(G)\right) \mid a, c \in \mathbb{N}\right\}$ is dense in the interval $\left(\frac{5}{6}, \frac{5}{3}\right)$. Observe that

$$
\delta\left(\hat{\varphi}_{c}^{a}(G)\right)= \begin{cases}\frac{20}{12} & \text { if } c=0 \\ \frac{19+20 a+10 c}{12(1+a+c)} & \text { if } c \neq 0,\end{cases}
$$

and suppose $\delta\left(\hat{\varphi}_{c}^{a}(G)\right)=\frac{r}{s}$ for some $\frac{r}{s} \in\left(\frac{5}{6}, \frac{5}{3}\right)$. Solving for $c$ in the case when $c>0$ we obtain

$$
c=\frac{(20 s-12 r) a+19 s-12 r}{12 r-10 s} .
$$

Notice that if $s$ is odd, then the numerator of this expression for $c$ is odd whereas the denominator is even, and thus this value of $c$ is not an integer. It follows that $s$ is even, so no rational number in reduced form with an odd denominator is $\delta\left(\hat{\varphi}_{c}^{a}(G)\right)$ for any values of $a$ and $c$. Finally, the reader may be entertained by the observation that both the set of GP distance numbers and non-GP distance numbers in $\left(\frac{5}{6}, \frac{5}{3}\right)$ are dense.

\section{ORCID iDs}




\section{References}

[1] A. R. Ashrafi and H. Shabani, The modified Wiener index of some graph operations, Ars Math. Contemp. 11 (2016), 277-284, doi:10.26493/1855-3974.801.968.

[2] D. Bonchev and N. Trinajstić, Information theory, distance matrix, and molecular branching, $J$. Chem. Phys. 67 (1977), 4517-4533, doi:10.1063/1.434593.

[3] R. Frucht, Herstellung von Graphen mit vorgegebener abstrakter Gruppe, Compositio Math. 6 (1939), 239-250, http: / /www. numdam.org/item?id=CM_1939_6_239_0.

[4] A. Graovac and T. Pisanski, On the Wiener index of a graph, J. Math. Chem. 8 (1991), 53-62, doi:10.1007/bf01166923, mathematical chemistry and computation (Dubrovnik, 1990).

[5] I. Gutman and T. Körtvélyesi, Wiener indices and molecular surfaces, Z. Naturforsch. 50 (1995), 669-671, doi:10.1515/zna-1995-0707.

[6] I. Gutman and O. E. Polansky, Wiener numbers of polyacenes and related benzenoid molecules, Match 20 (1986), 115-123, https : / match.pmf.kg.ac.rs/content20 . htm.

[7] M. A. Iranmanesh and H. Shabani, The symmetry-moderated Wiener index of truncation graph, Thorn graph and caterpillars, Discrete Appl. Math. 269 (2019), 41-51, doi:10.1016/j.dam.2018. 05.040 .

[8] M. Knor, J. Komorník, R. Škrekovski and A. Tepeh, Unicyclic graphs with the maximal value of Graovac-Pisanski index, Ars Math. Contemp. 17 (2019), 455-466, doi:10.26493/1855-3974. 1925.57a.

[9] M. Knor, R. Škrekovski and A. Tepeh, Chemical graphs with the minimum value of Wiener index, Match Commun. Math. Comput. Chem. 81 (2019), 119-132, https: / / match.pmf . kg.ac.rs/content81n1.htm.

[10] M. Knor, R. Škrekovski and A. Tepeh, Mathematical aspects of Wiener index, Ars Math. Contemp. 11 (2016), 327-352, doi:10.26493/1855-3974.795.ebf.

[11] B. Mohar and T. Pisanski, How to compute the Wiener index of a graph, J. Math. Chem. 2 (1988), 267-277, doi:10.1007/bf01167206.

[12] R. Pinal, Effect of molecular symmetry on melting temperature and solubility, Org. Biomol. Chem. 2 (2004), 2692-2699, doi:10.1039/b407105k.

[13] H. Wiener, Correlation of heats of isomerization, and differences in heats of vaporization of isomers, among the paraffin hydrocarbons, J. Am. Chem. Soc. 69 (1947), 2636-2638, doi: $10.1021 / \mathrm{ja} 01203 \mathrm{a} 022$.

[14] H. Wiener, Structural determination of paraffin boiling points, J. Am. Chem. Soc. 69 (1947), 17-20, doi:doi.org/10.1021/ja01193a005. 